\title{
Upper Body of a new Humanoid Robot - the Design of ARMAR III
}

\author{
Albert Albers, Sven Brudniok, Jens Ottnad, Christian Sauter, Korkiat Sedchaicharn \\ Institute of Product Development \\ University of Karlsruhe (TH) \\ Karlsruhe, Germany \\ \{albers, brudniok, ottnad, sauter, sedchaicharn\}@ipek.uni-karlsruhe.de
}

\begin{abstract}
The development of a humanoid robot platform within the scope of the collaborative research centre 588 has the objective of creating a machine that closely cooperates with humans. This development area presents a new challenge to designers. In contrast to industrial robots - for which mechanical rigidity, precision and high velocities are primary requirements the key aspects here are prevention of hazards to users, a motion space that corresponds to that of human beings and a lightweight design. In order to meet these requirements, the robot must have humanlike appearance, motion space and dexterity. Additionally, its kinematics should be familiar to the user, its motions predictable, so as to encourage inexperienced persons to interact with the machine.

This article gives insight into the design of the mechatronic components of the upper body of the humanoid robot ARMAR III. Due to the special boundary conditions for the design of such a humanoid robot and complex interaction between system elements, the design process is very challenging. The robot has a modular structure. The modules for neck, torso and arms were designed and built at the Institute of Product Development (IPEK) at the University of Karlsruhe (TH). The design of these modules of the upper body and the problems solved by these designs are presented in this article.
\end{abstract}

Index Terms - Humanoid Robot; design.

\section{INTRODUCTION}

The mechatronic design of a humanoid robot is fundamentally different from that of industrial robots. Industrial robots generally have to meet requirements like mechanical stiffness, accuracy and high velocities. The key goal for this humanoid robot is not accuracy, but the ability to cooperate with humans. In order to enable a robot to interact with humans, high standards are set for sensory senses for controlling these movements. Its kinematic properties and its range of movements have to be adjusted to humans and their environment [1].

The collaborative research centre 588 "Humanoid Robots learning and cooperating multi-modal robots" was established by the "Deutsche Forschungsgemeinschaft" (DFG) in Karlsruhe in May 2001. In this project, scientists from different academic fields develop concepts, methods and concrete mechatronic components for a humanoid robot that can share its working space with humans. The long-term target is the interactive work of robots and humans for example in the kitchen.

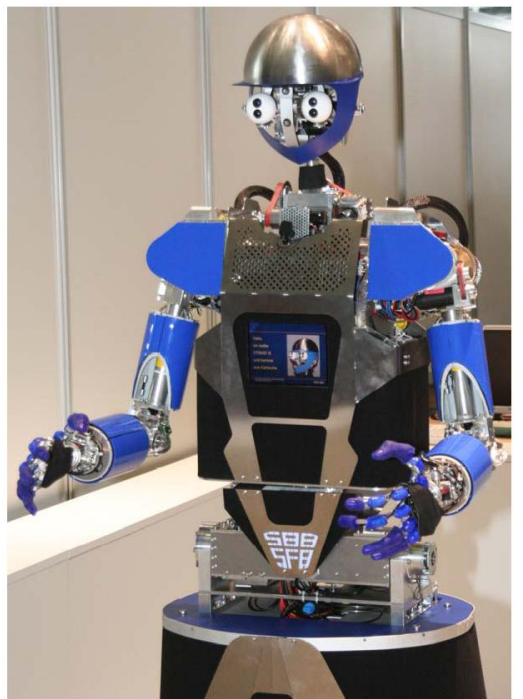

Fig. 1 ARMAR III

For instance a simple task like putting dishes into a dishwasher requires sophisticated skills in cognition and the manipulation of objects. Communication between robots and humans should be possible in different ways, like speech, touch or gestures, making an intuitive handling easier for the human. As this is the main focus of the collaborative research centre a humanoid upper body on a holonomic platform for locomotion has been developed. It is planned to increase the mobility of ARMAR by replacing the platform by legs in a future product generation, which will lead to modifications at the upper body.

The robot ARMAR III was presented to the public at the computer fair CEBIT in spring 2006 in Hannover. Most mechatronic modules have been designed and built at the Institute of Product Development (IPEK) at the University of Karlsruhe (TH). The results of this works are presented in this article.

\section{TARGET SYSTEMS AND DEMANDS}

Since the robot is to get into contact with humans in order to fulfill various functions, it is important that the robot is accepted by the human. A human-like appearance is as important as the ability to move like the human. Specific demands [2] on kinematics, dynamics and the design space 
have to be considered. An example for one of these requirements is that all rotational axes of one joint intersect in one common point. At the same time a good control is wanted in order to run all degrees of freedom as individually as possible.

As actuators only servomotors are used, in order to provide a standardized drive concept. The drive units are dimensioned for a load of $3 \mathrm{~kg}$. All gears are designed to be not selflocking, but friction leads, e.g. in case of a loss of power, to a slow and damped sinking of the arm instead of abrupt movement. That is of great importance for an interactive application of the robot in a human environment. On the other hand, stick-slip effects in the gears have been avoided, which is a clear benefit for the control system.

In order to realize the abilities mentioned above, different types of sensors are necessary in every degree of freedom. The goal was the integration of an angular position measurement with an encoder on the motor, an absolute angular position measurement close to the joint and a measuring of the forces in the drive train.

For energy efficient operation of an autonomous system, it is necessary to achieve a lightweight design. It is especially important to design these components as light as possible which are moved in highly dynamic motions and which contribute mainly to the relevant inertia of a module. Also regarding the security of the user, for example in case of collisions, which can happen during performing common activities with the robot, this is of importance.

Some of these requirements, like accuracy for the positioning of an end-effector and a structure that is as light as possible, are contrary design goals. This is only one of the problems in the design of a humanoid robot structure that demonstrates the difficulty of such a design task.

\section{UPPER BODY}

The robot ARMAR III has a total of 45 degrees of freedom and can be divided into a humanoid upper body and a holonomic platform for locomotion. The upper body consists of the following modules: two arms with shoulder, elbow, wrist and hands, head, neck joint, thorax and torso joint. The head and the holonomic platform were developed at the "Forschungszentrum Informatik" (FZI) and the hands by the "Institut für Angewandte Informatik" in Karlsruhe [3],[4]. The remaining modules, which are presented in this article, were created at the "Institute of Product Development".

The kinematics of these modules and a CAD model are shown in fig. 2.

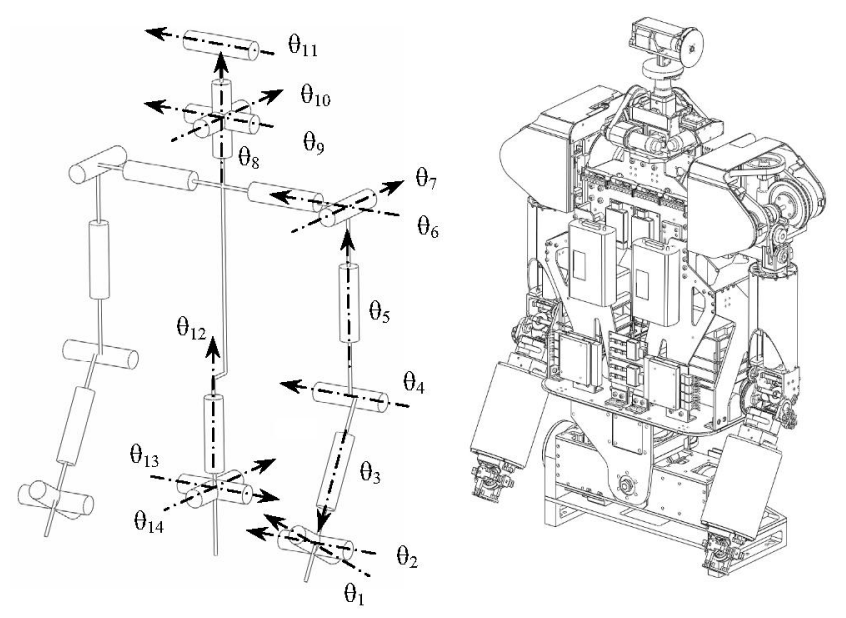

Fig. 2. Kinematics and CAD model of upper body

The size of the design space and the motion space of ARMAR III are similar to that of a human person with a height of approximately $175 \mathrm{~cm}$. The most important dimensions of the upper body can be seen in fig. 3 .

Table 1 gives an overview of all modules with the corresponding degrees of freedom and the motion range. Both arms have seven degrees of freedom. The three degrees of freedom in the shoulder provide a relatively wide range of motion. Together with two degrees of freedom in the elbow as well as in the wrist, the arm can be used for complex manipulation tasks that may occur in the primary working environment of ARMAR III, the kitchen. Compared with other humanoid robots, the arm of ARMAR III provides one of the largest and most humanlike ranges of motion. Apart from the fact that the neck joint with four degrees of freedom contributes significantly to the humanlike motion of the head.

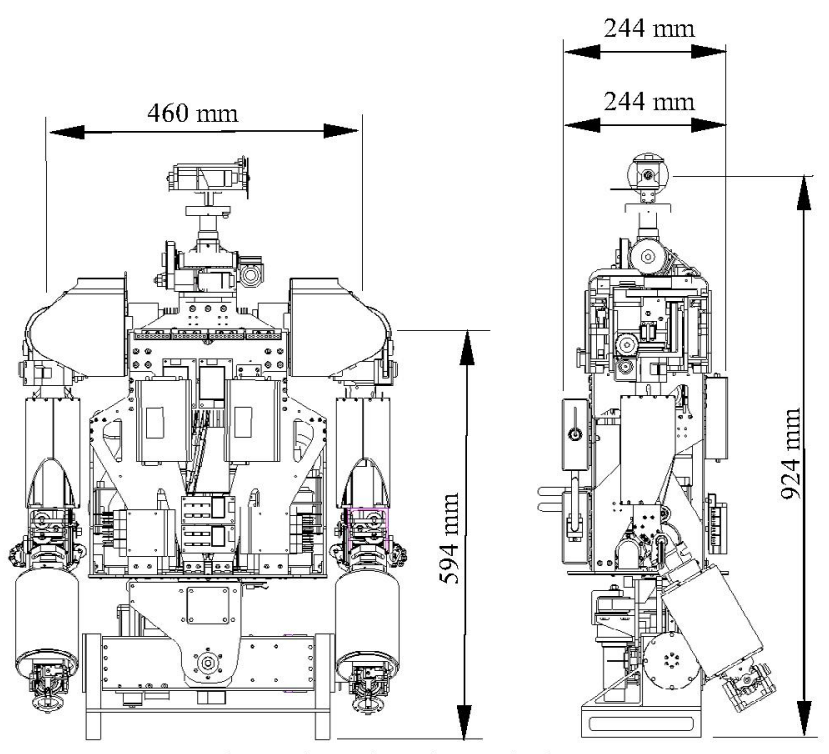

Fig. 3 Dimension of upper body 


\begin{tabular}{|c|c|c|c|c|c|}
\hline \multirow[t]{7}{*}{ Degree of freedom } & \multicolumn{2}{|l|}{ Part } & D.O.F & amount & total \\
\hline & \multicolumn{2}{|l|}{ Wrist } & 2 & 2 & 4 \\
\hline & \multicolumn{2}{|l|}{ Elbow } & 2 & 2 & 4 \\
\hline & \multicolumn{2}{|l|}{ Shoulder } & 3 & 2 & 6 \\
\hline & \multicolumn{2}{|l|}{ Neck } & 4 & 1 & 4 \\
\hline & \multicolumn{2}{|l|}{ Torso } & 3 & 1 & 3 \\
\hline & \multicolumn{4}{|c|}{ Upper body } & 21 \\
\hline \multirow[t]{13}{*}{ Movable range } & \multirow[t]{2}{*}{ Wrist } & $\theta_{1}$ & \multicolumn{3}{|c|}{$-30^{\circ}$ to $30^{\circ}$} \\
\hline & & $\theta_{2}$ & \multicolumn{3}{|c|}{$-60^{\circ}$ to $60^{\circ}$} \\
\hline & Elbow & $\begin{array}{l}\theta_{3} \\
\theta_{4}\end{array}$ & \multicolumn{3}{|c|}{$-90^{\circ}$ to $90^{\circ}$} \\
\hline & \multirow{3}{*}{ Shoulder } & $\theta_{5}$ & \multicolumn{3}{|c|}{$-180^{\circ}$ to $180^{\circ}$} \\
\hline & & $\theta_{6}$ & \multicolumn{3}{|c|}{$-45^{\circ}$ to $180^{\circ}$} \\
\hline & & $\theta_{7}$ & \multicolumn{3}{|c|}{$-10^{\circ}$ to $180^{\circ}$} \\
\hline & \multirow[t]{4}{*}{ Neck } & $\theta_{8}$ & \multicolumn{3}{|c|}{$-180^{\circ}$ to $180^{\circ}$} \\
\hline & & $\theta_{9}$ & \multicolumn{3}{|c|}{$-45^{\circ}$ to $45^{\circ}$} \\
\hline & & $\theta_{10}$ & \multicolumn{3}{|c|}{$-45^{\circ}$ to $45^{\circ}$} \\
\hline & & $\theta_{11}$ & \\
\hline & Torso & $\theta_{12}$ & \multirow{3}{*}{\multicolumn{3}{|c|}{$\begin{array}{l}-180^{\circ} \text { to } 180^{\circ} \\
-10^{\circ} \text { to } 60^{\circ} \\
-20^{\circ} \text { to } 20^{\circ}\end{array}$}} \\
\hline & & $\theta_{13}$ & & & \\
\hline & & $\theta_{14}$ & & & \\
\hline
\end{tabular}

Table 1. Specifications of parts of upper body without hands and head

\section{SHOULDER JOINT}

The shoulder joint is the link between the arm and the torso. In addition to the realization of three degrees of freedom with intersecting axes in one point, the bowden cables for driving the elbow joint must be guided through the shoulder joint from the elbow drive units in the torso to the elbow. The drive units of all joints are designed in such a way, that their contributions to the inertia are as small as possible. For this reason the drive unit for panning the arm (Rot. 1), which has to provide the highest torque for the arm, is attached directly to the torso and therefore does not contribute to the inertia of the arm. The drive units for raising the arm (Rot. 2) and turning the arm around its longitudinal axis (Rot. 3) have been placed closely to the rotational axes to improve the dynamics of the shoulder joint. In order to achieve the required gear ratios in the very limited design space, Harmonic Drive transmissions, worm gear transmissions and toothed belt transmissions have been used.

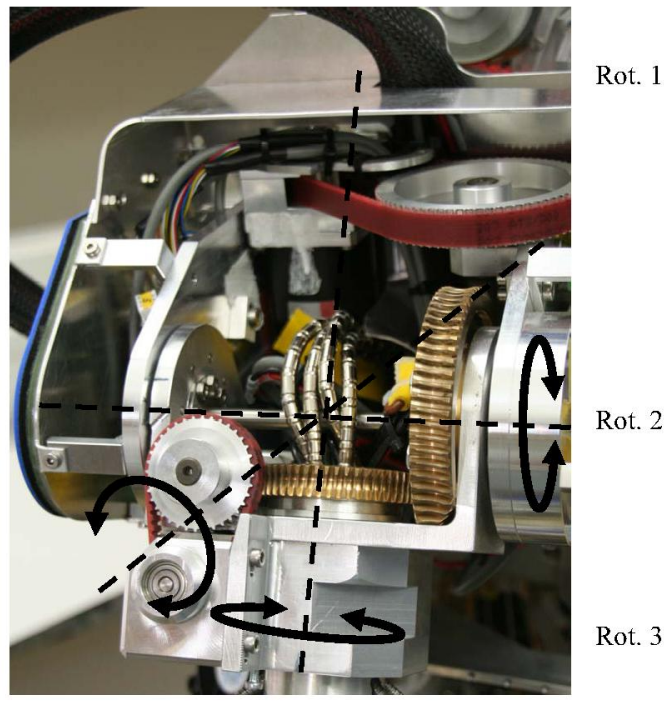

Fig. 4 View of the shoulder joint from the side
These elements allowed for a compact design of the shoulder with a size similar to a human shoulder. As all degrees of freedom are realized directly in the shoulder, the upper arm of the design is slender. Compared to the arm of the version II, the space required for mounting the shoulder was reduced by approximately $50 \%$, which allowed for the integration of two computers in the torso.

The integration of torque sensors in all three degrees of freedom is realized in two different ways. For the first degree of freedom strain gages are attached to a torsion shaft that is integrated in the drive train. The torque for raising and turning the arm is determined by force sensors that measure the axial forces in the worm gear shafts.

In addition to the encoders, which are attached directly at the motors, angular sensors for all three degrees of freedom are integrated into the drive trains of the shoulder joints. The position sensors, which are located directly at the joints, allow quasi-absolute angular position measurement based on incremental optical sensors [5]. A touch-sensitive artificial skin sensor is attached to the front and rear part of the shoulder casing [6]. This gives the user the possibility to communicate with the robot by touch, for instance the robot can be directed by pushing or pulling the shoulders or the user can get the robots attention by tapping its shoulder.

\section{ELBOW JOINT AND UPPER ARM}

The elbow joint of ARMAR III has two degrees of freedom. Thus bending as well as a rotation of the forearm is possible. The drive units, consisting of motor and Harmonic Drive transmissions, are not in the arm, but are located in the thorax of the robot. Thus the mass as well as the necessary design space of the arm are strongly reduced, which leads to better dynamic characteristics and a slim form of the arm. The additional mass in the thorax contributes substantially less to the mass inertia than when the drive units are placed in the arm. Due to this concept load transmission is implemented

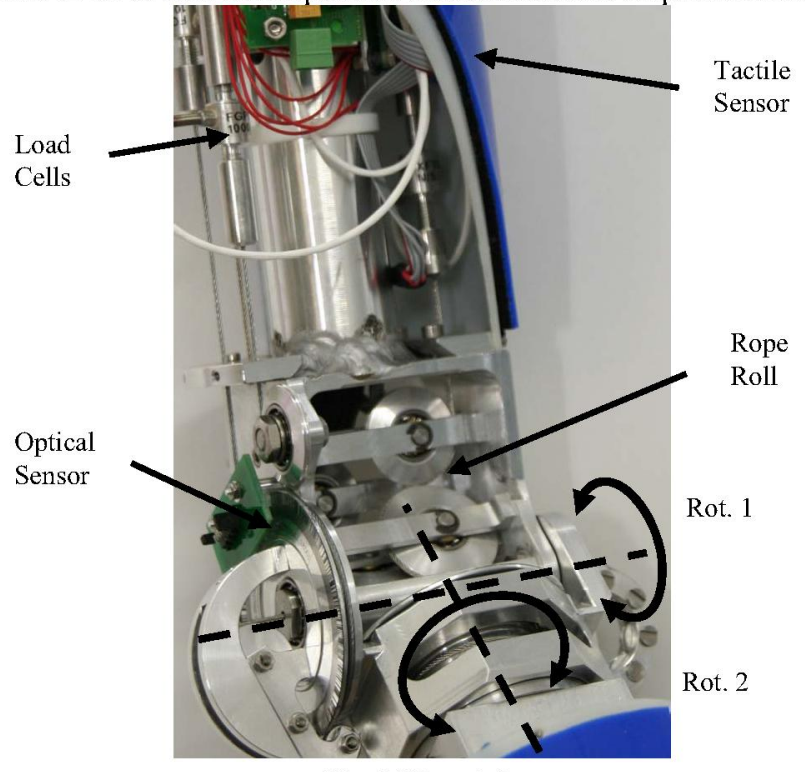

Fig. 5 Elbow joint 
with the use of wire ropes, which are led from the torso through the shoulder to the elbow by rolls and bowden cables. In order to make an independent control possible of the two degrees of freedom, the wire ropes for the turn of the forearm are led through the axis of rotation for bending of elbow. With altogether twelve rolls this rope guide realizes the uncoupling of the motion of bending the elbow from rotating the forearm. In contrast to the elbow version II, this solution leads to small and constant friction losses which in particular provides advantages for the force control. Furthermore a compact design was achieved.

Exactly as in the shoulder the angular measurement is accomplished by encoders attached directly to the motors as well as optical sensors that are located directly at the joint for both degrees of freedom. In order to measure the drive torque, load cells are integrated in the wire ropes in the upper arm. As each degree of freedom in the elbow is driven by two wire ropes the measuring of force in the wire ropes can be done by difference measurements, whereby influences on the measurement results, such as drifts in temperature, are not taken into account. A further possibility for the measurement of forces offers the tactile sensor skin, which is integrated in the casing of the upper arm.

By placing the drive units in the thorax, there is enough free space left in the arm. Therefore the electronic components for processing the sensor signals can be installed in direct proximity to the sensors in the upper arm.

\section{WRIST JOINT AND FOREARM}

The wrist has two degrees of freedom. Its rotational axes intersect in one point. ARMAR III has the ability to move the wrist to the side as well as up and down. This was realized by universal joint in very compact construction. The motors for both degrees of freedom are fixed at the support structure of the forearm. The gear ratio is obtained by a ball screw and a toothed belt or a wire rope. The load transmission is almost free from backlash. Compared to the wrist joint of the version II, significant progress was achieved. By arranging

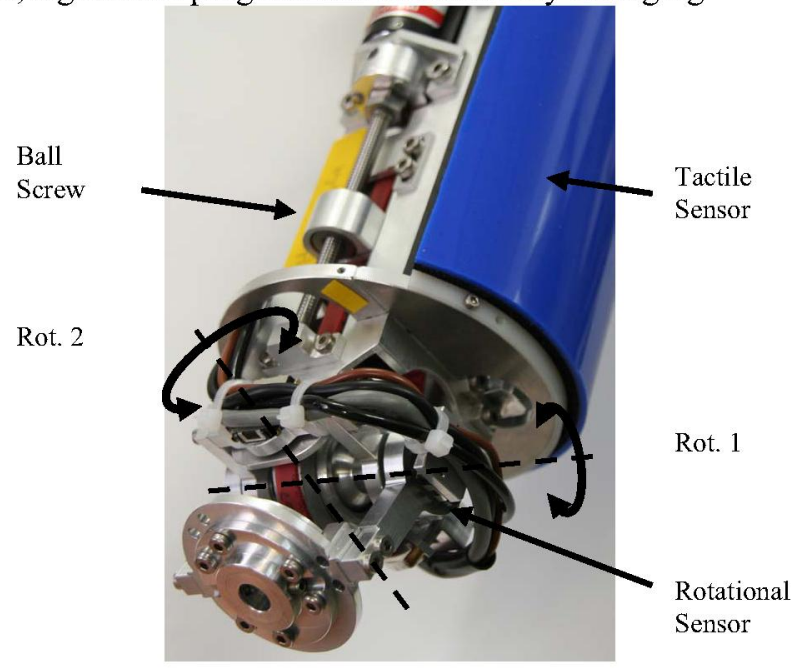

Fig. 6 Forearm with two degrees of freedom in the wrist the motors close to the elbow joint, the centre of mass of the forearm is shifted towards the body, which is an advantage for typical movements of the robot.

The angular measurement in the wrist is realized by encoders at the motors and with quasi-absolute angular sensors directly at the joint. To measure the load on the hand, a 6-axis force and torque sensor is fitted between the wrist and the hand [3] (not shown in fig.6). The casing of the forearm is also equipped with a tactile sensor skin.

The support structure of the forearm consists of a square pipe. This rigid lightweight structure offers the possibility of cable routing on the inside and enough space for electronic components on the exterior at the same time.

\section{NECK JOINT}

The complex kinematics of the human neck is defined by seven cervical vertebrae. Each connection between two vertebrae can be seen as a joint with three degrees of freedom. For this robot the kinematics of the neck has been reduced to a serial kinematics with four rotational degrees of freedom. Three degrees of freedom were realized in the basis at the lower end of the neck. Two degrees of freedom allow the neck to lean forwards and backwards (1) and to the side (2), another degree of freedom allows rotation around the longitudinal axis of the neck. At the upper end of the neck a fourth degree of freedom allows nodding of the head. This degree of freedom allows more human-like movements of the head and improves the robots ability to look up and down and to detect objects directly in front of it.

For the conversion of torque and rotational speed, the drive train of each degree of freedom consists of Harmonic Drive transmissions either as only transmission element or, depending on the needed overall gear ratio, in combination with a toothed gear belt. With these concepts, the drives for all degrees of freedom in the neck are practically free from backlash. The motors of all degrees of freedoms are placed as close as possible to the rotational axis in order to keep the moment of inertia small. The sensors for the angular position measurement in the neck consist of a combination of

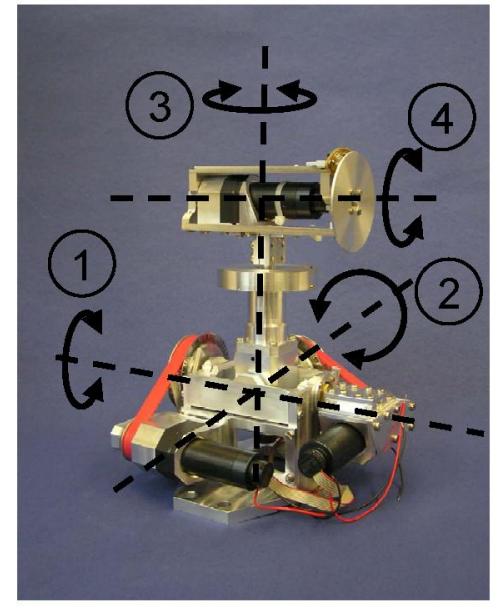

Fig. 7 Neck joint with four degrees of freedom 
incremental encoders, which are attached directly to the motors, and quasi-absolute optical sensors, which are placed directly at the rotational axis.

\section{THORAX}

The thorax of ARMAR III is different from the other parts of the upper body. The thorax has no degree of freedom. For this reason, its design will be focused on the topology optimization and the arrangement of mechanical and electrical components, which must be assembled in the thorax. For instance the four drive units for the elbows have to be integrated in the thorax to decrease the weight of the arms. The electrical components for the upper body, such as two PC-104s, four Universal Controller Modules (UCoM), A/D converter, $\mathrm{DC} / \mathrm{DC}$ converters and force-moment controllers, are also considered for the spatial arrangement.
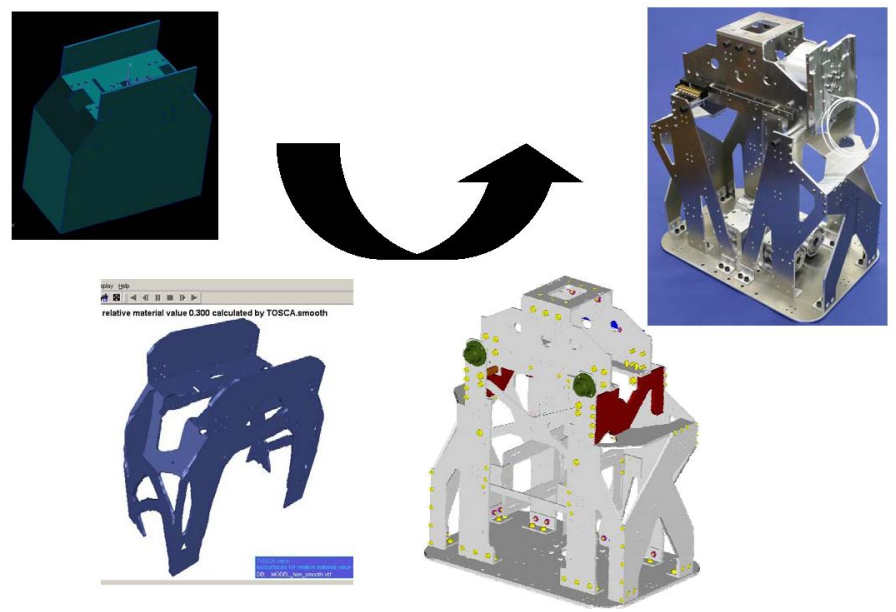

Fig. 8. Topology optimization of the thorax

The design of the thorax was determined by computer aided topology optimization and consists of high-strength aluminum plates. Not only topology optimization, but also the arrangement of components is important for the thorax design. All components were modeled and iteratively positioned in CAD model to obtain an optimized location for each component. The result of the optimization is a lightweight structure with a weight of only $2.7 \mathrm{~kg}$. It is shown in fig. 8 . In the figure it can be seen that all four drive units, that are relatively heavy, are located at the bottom of the thorax to minimize the necessary torque in torso joint. All components are arranged symmetrically to balance the weight between the left and the right side of the upper body.

\section{TORSO JOINT}

The torso of the upper body of ARMAR III is divided in two parts, the thorax and the torso joint below it. The torso joint allows motion between the remaining upper body relatively and the holonomic platform, similar to the functionality given by the lower back and hip joints in the human body. The kinematics of this torso joint does not exactly replicate the complex human kinematics of the hip joints and the lower back. The complexity was reduced in consideration of the functional requirements which result from the main application scenario of this robot in the kitchen. The torso joint has three rotational degrees of freedom with the axes intersecting in one point. The kinematics of this joint, as it is described in table 1 and fig. 9, is sufficient to allow the robot to easily reach important places in the kitchen. For example in a narrow kitchen, the whole upper body can turn sideways or fully around without having to turn the platform. One special requirement for the torso joint is, that all cables for the electrical energy flow and information flow between the platform and the upper body need to go through the torso joint. All cables are to be led from the upper body to the torso joint in a hollow shaft with an inner bore diameter of $40 \mathrm{~mm}$ through the point of intersection of the three rotational axes. This significantly complicates the design, but the cable connections can be shorter and stresses on the cables due to compensational motions, that would be necessary if the cable routing was different, can be avoided. This simplifies the design of the interface between upper and lower body. For transportation of the robot these two parts can be separated by loosening one bolted connection and unplugging a few central cables.

In the torso joint also electronic motors are used as actuators for a homogenous drive and control concept. Due to the special boundary conditions from the cable routing, all motors had to be placed away from the crossing point of the three axes and the motor for the vertical degree of freedom (Rot.3) could not be positioned coaxially to the axis of rotation. The drive train for the degrees of freedom Rot. 1 and Rot. 3 consists of Harmonic Drive transmissions and toothed belt transmissions. The drive train for the degree of freedom Rot.2 is different from most of the other drive trains in ARMAR III as it consists

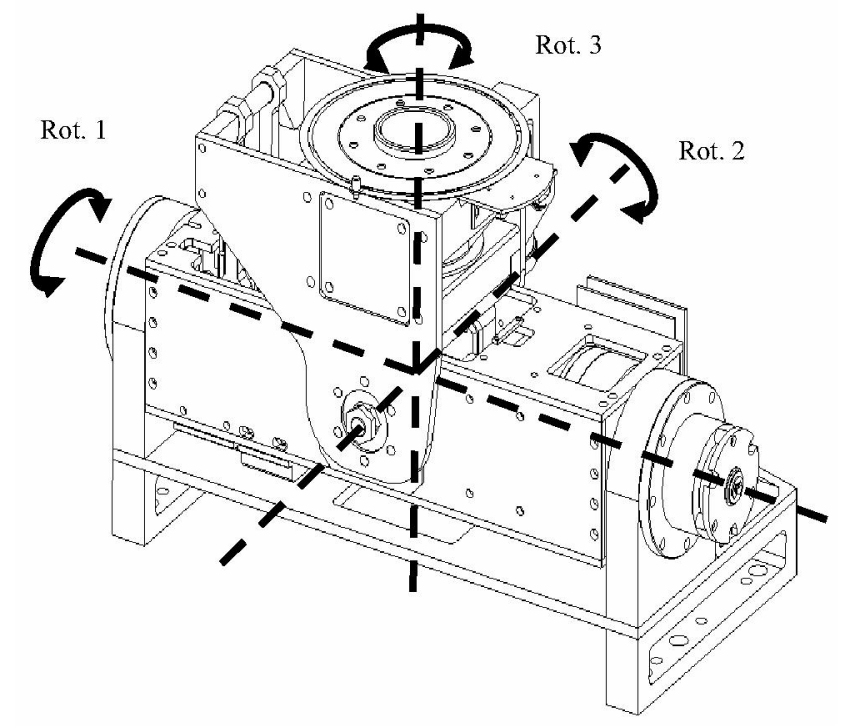

Fig. 9.Torso Joint

of a toothed belt transmission, a ball screw and a piston rod which transforms the translational motion of the ball screw 
into the rotational motion for moving the upper body sideways. This solution is suitable for the relatively small range of motion of $40^{\circ}$ for this degree of freedom and was chosen because a high gear ratio can be achieved and the motor can be placed away from the driven axis and away from the point of intersection of the rotational axes.

In addition to the encoders, which are directly attached to the motors, 2 precision potentiometers and one quasi-absolute optical sensor are used for the angular position measurement.

\section{ACKNOWLEDGMENT}

The work presented in this article is funded by the "Deutsche Forschungsgemeinschaft" (DFG).

\section{REFERENCES}

[1] C. Schäfer: Entwurf eines anthropomorphen Roboterarms: Kinematik, Arbeitsraumanalyse, Softwaremodellierung, Dissertation Fakultät für Informatik, Universität Karlsruhe, 2000.

[2] T. Asfour: Sensomotorische Bewegungskoordination zur Handlungsausführung eines humanoiden Roboters, Dissertation Fakultät für Informatik, Universität Karlsruhe, 2003.

[3] S. Beck, A. Lehmann, Th. Lotz, J. Martin, R. Keppler, R. Mikut: Modelbased adaptive control of a fluidic actuated robotic hand, Proc., GMAKongress 2003, VDI-Berichte 1756, S. 65-72; 2003.

[4] S. Schulz: Eine neue Adaptiv-Hand-Prothese auf der Basis flexibler Fluidaktoren, Dissertation, Fakultät für Maschinenbau, Universität Karlsruhe (TH), 2003

[5] O. Kerpa, K. Weiss, H. Wörn: Development of Flexible Tactile Sensor System for a Humanoid Robot, Intelligent Robots and Systems, 2003. (IROS 2003)

[6] ATI Industrial Automation, catalog 2002. 